\title{
Autophagy in protozoan parasites: Trypanosoma brucei as a model
}

\author{
Feng-Jun $\mathrm{Li}^{*}, 1$ \& Cynthia $\mathrm{Y} \mathrm{He}{ }^{* *}, 2$ \\ ${ }^{1}$ Department of Microbiology \& Immunology, YLL School of Medicine, National University of Singapore, 5 Science Drive 2, \\ Singapore 117545 \\ ${ }^{2}$ Department of Biological Sciences, National University of Singapore, 14 Science Drive 4, Singapore 117543 \\ * Author for correspondence: Tel.: +65 9389 3127; miclfj@nus.edu.sg \\ ** Author for correspondence: Tel.: +65 6516 7377; dbshyc@nus.edu.sg
}

"To gain further and more complete understanding of the mechanisms and functions of autophagy, both conserved and evolutionarily divergent, study of more divergent model systems is needed."

First draft submitted: 24 July 2017; Accepted for publication: 18 August 2017; Published online: 3 October 2017

Keywords: autophagy $\bullet$ protozoan parasite $\bullet$ Trypanosoma brucei

Autophagy is an intracellular pathway that clears macromolecules, organelles and intracellular pathogens through autophagosomes and lysosomes upon nutrient limitation, infections and other physiological/pathological conditions. Autophagy has been best studied in budding yeast and mammals, revealing highly conserved machineries and physiological importance in development and diseases. In general, autophagy is triggered upon inhibition of TOR, or activation of AMPK; both conserved protein kinases. Phosphorylation and activation of ATG13 lead to activation of ULK1/2 (Atg1 in yeast), which further stimulates autophagic modules including the PtdIns3K complex: VPS34 and Beclin1, and the unbiquition-like conjugation system that includes ATG8 and ATG12. VPS34 produces Pt$\mathrm{dIns} 3 \mathrm{P}$ at certain cellular locations, which serves as a signal to recruit other proteins for phagophore formation. The phagophores elongate and engulf cytoplasmic components, forming double-membraned autophagosomes. Finally, autophagosomes are transported to and fused with lysosomes, where the engulfed materials are degraded.

Most of the autophagy-related genes identified are highly conserved functionally in yeast and mammals, although different regulatory or signaling mechanisms have been found for some molecules, mTOR as an example [1]. The protozoan parasites are evolutionarily divergent, single-celled eukaryotic pathogens including the Plasmodium spp. that causes malaria; Toxoplasma gondii that causes toxoplasmosis; and Trypanosoma brucei, Trypanosoma cruzi and Leishmania spp. that cause the tritryp diseases. Together these pathogens cause huge economic and disease burdens globally. Bioinformatics analysis identified only about half of the ATG homologs in these protozoan parasites [2], implying a simple or divergent autophagy pathway in these organisms. To gain further and more complete understanding of the mechanisms and functions of autophagy, both conserved and evolutionarily divergent, study of more divergent model systems is needed.

\section{Advantages of $T$. brucei as a model organism for cell biology studies}

Among the protozoan parasites, T. brucei is one of the most genetically accessible for study in laboratories. Like many parasites, T. brucei undergoes a complex life cycle in the insect vector and mammalian hosts, taking on different developmental forms. Cultivation and differentiation of some life-cycle stages can be achieved in vitro, making $T$. bruce $i$ an ideal organism to study the signaling and cellular remodeling mechanisms during life-cycle differentiation and development. Advanced reverse genetics methods such as inducible expression [3,4] and RNAi [5] allow rapid characterization of protein functions. Additionally, T. brucei possesses a variety of organelles, ubiquitously found in all eukaryotes or uniquely present in trypanosomes, many of them present at single copy and occupy a distinct location in the highly polarized cell. Thus T. brucei has also been useful for cell biology research to study organelle biogenesis and protein trafficking. 
In response to concerns that the biology in this lower eukaryote is too divergent to the 'crown' model organisms, Graham Warren, an eminent cell biologist gives his view [6], which we strongly agree: “. . . So it seems to me to make far more sense to use whatever system can best answer the questions that most fascinate you, even if it means straying beyond the confines of the crown eukaryotes."

\section{Autophagy in T. brucei}

Autophagy research involving parasitic pathogens mainly focus on host-autophagic responses to infections, and how pathogens exploit or evade host autophagy for survival. These studies revealed important functions of host autophagy in inflammations and immunity and complexity of host-pathogen interactions [7]. Recent studies confirm the occurrence of autophagy during nutrient starvation and life-cycle developments in several parasites. Starvation-, endoplasmic reticulum stress- and drug-induced autophagy-like cell death have also been observed in T. brucei, T. cruzi, Leishmania donovani, Toxoplasma gondii and Plasmodium falciparum, suggesting a prodeath function of autophagy in these parasites [8,9]. Although the role of autophagy in parasite-programmed cell death remains controversial, and whether it contributes to parasite population control in specific life stage(s) remains unclear, it is widely accepted that autophagy has a tight association with organelle turnover, nutrient utilization and metabolism in these parasites [10,11]. The bona fide occurrence of autophagy in the protozoan pathogens thus identified an emerging requirement and opportunity to explore the physiological triggers and functions of parasite autophagy during animal infections.

At the molecular level, the ATG8-phosphatidylethanolamine conjugation system is highly conserved while proteins involved in many other autophagy steps lack conservation or are completely missing in the parasite genomes [2]. In T. gondii and P. falciparum, ATG8 localizes to the apicoplast, a residual plastid, under both normal and nutrient-limit conditions, suggesting an unusual link between autophagy and apicoplast biogenesis [12]. The persistent apicoplast localization of ATG8 and the lack of other bona fide autophagy markers make autophagy study challenging in these parasites. In T. brucei, using ATG8 homolog TbATG8.2 as marker, autophagosome formation can be reliably monitored in vitro during starvation and chemical treatments. The core autophagy events, from double-membraned autophagosome formation to the final degradation in lysosomes are conserved [8], and autophagy-related functions of several ATG homologs or protein complex including ATG3, ATG5, ATG7, ATG24 and PI3K have also been confirmed [8,13-14]. Interestingly, the acidocalcisomes, a lysosome-related organelle found in trypanosomes and some other protozoan parasites are found to be required for autophagy induction [15]. In higher eukaryotes, the lysosomes are not only involved in the last degradation step of autophagy, but also involved in the induction step through mTOR signaling. In T. brucei, however, the induction signaling and degradation functions appear to be separately governed by two organelles, induction signaling via the acidocalcisomes and degradation via the lysosomes. Further studies of the molecular details of autophagy signaling in parasitic protozoa will undoubtedly enhance our understanding of the diversity and complexity of autophagy and the opportunities it may offer as a drug target to control these important parasites.

The physiological trigger and relevance of parasite autophagy during animal infections remain obscure. In mammal cells, autophagy can be triggered by low cellular energy charge or deprivation of essential nutrients, including glucose and amino acids [16]. The AMPK can sense low energy level (high AMP/ATP ratio) in cells that usually occurs upon glucose depletion, and acts as a checkpoint for cell growth, autophagy and metabolism coordination. On the other hand, limitations of nonessential amino acids trigger autophagy by inactivation of mTORC1. T. brucei typically resides in the bloodstream of the mammalian hosts (as the bloodstream form) or the midgut of the tsetse fly (as the procyclic form), both considered as nutrient-rich environments. Does T. brucei autophagy occur in vivo during infections?

The bloodstream form T. brucei generates ATP exclusively through glycolysis of glucose, which is abundantly present in the blood of the vertebrate hosts. In the procyclic form, the parasite mitochondrion is fully developed, and ATP can be produced by oxidative phosphorylation in addition to glycolysis. Additionally, proline, which is an abundant energy source in the tsetse fly, is also key energy source for procyclic trypanosomes since glucose is scarce in the tsetse midgut. However, when cultivated in medium with glucose, the parasites prefer glucose by inhibiting the proline uptake and proline dehydrogenase activity, and to generate ATP by glycolysis [17]. Utilizing these unique features of T. brucei metabolism, we show that autophagy activity in T. brucei depends on intracellular ATP level. While T. brucei autophagy can be robustly triggered by amino acid starvation, low cellular energy charge (achieved by removing carbon sources or inhibiting ATP production pathways) cannot induce autophgy and an AMPK-mediated energy sensing mechanism is not involved in trypanosomal autophagy [18]. 
Previous metabolic studies of $T$. brucei-infected mice demonstrate the depletion of several amino acids but relatively stable glucose level in plasma as infection progresses and parasitemia increases. Our unpublished work show increased autophagosome formation in the parasites late in infection at high parasitemia, likely induced by depleted plasma amino acids and fueled by persistent plasma glucose. This ATP-driven and AMPK-independent autophagy may thus represent a specialized adaptation for the parasitic life style of $T$. brucei, facilitating cell differentiation at high parasitemia. Further understanding of the specialized autophagy regulation and signaling pathways in T. brucei will be an important next step, taking advantage of the powerful molecular genetics toolkits and the recently developed genome-wide RNAi screening method.

\section{What can T. brucei offer to the general understanding of autophagy?}

Although autophagy is a highly conserved process, functional and regulatory variations are observed. For example, ATG5/ATG7-independent autophagy occurs in mouse cells when subjected to certain stresses [19]. How common are the noncanonical autophagic mechanisms and what is the physiological relevance of having multiple autophagy control mechanisms are still open questions in the autophagy community. Besides ATG5 and ATG7, ULK1 and PI3K complexes are known to be essential for the initiation of starvation-induced autophagy. A recent bioinformatics study found that the ULK1/ATG1 of protozoan parasites (including T. brucei ULK1/ATG1) lacks the activity domain and therefore unlikely to initiate autophagy induction through the ULK1/ATG1 pathway [20]. The divergence and the absence of several functional ATG homologs in T. brucei and other protozoan parasites make them important and interesting systems to possible noncanonical pathways. From a synthetic biology point of view, these 'simpler' eukaryotes may also allow 'engineering' or reconstitution of the more complex autophagy process as found in higher eukaryotes.

\section{Financial \& competing interests disclosure}

This work was funded by a Singapore Ministry of Education Tier 1 research grant awarded to CY He. The authors have no other relevant affiliations or financial involvement with any organization or entity with a financial interest in or financial conflict with the subject matter or materials discussed in the manuscript apart from those disclosed.

No writing assistance was utilized in the production of this manuscript.

\section{References}

1. Gonzalez A, Hall MN. Nutrient sensing and TOR signaling in yeast and mammals. EMBO J. 36(4), 397-408 (2017).

2. Duszenko M, Ginger ML, Brennand A et al. Autophagy in protists. Autophagy 7(2), 127-158 (2011).

3. Wirtz E, Clayton C. Inducible gene expression in trypanosomes mediated by a prokaryotic repressor. Science 268(5214), 1179-1183 (1995).

4. Li FJ, Xu ZS, Aye HM et al. An efficient cumate-inducible system for procyclic and bloodstream form Trypanosoma brucei. Mol. Biochem. Parasitol. 214, 101-104 (2017).

5. Ngo H, Tschudi C, Gull K, Ullu E. Double-stranded RNA induces mRNA degradation in Trypanosoma brucei. Proc. Natl Acad. Sci. USA 95(25), 14687-14692 (1998).

6. Warren G. In praise of other model organisms. J. Cell Biol. 208(4), 387-389 (2015).

7. Deretic V, Saitoh T, Akira S. Autophagy in infection, inflammation and immunity. Nat. Rev. Immunol. 13(10), 722-737 (2013).

8. Li FJ, Shen Q, Wang C, Sun Y, Yuan AY, He CY. A role of autophagy in Trypanosoma brucei cell death. Cell. Microbiol. 14(8), 1242-1256 (2012).

9. Sinai AP, Roepe PD. Autophagy in Apicomplexa: a life sustaining death mechanism? Trends Parasitol. 28(9), 358-364 (2012).

10. Herman M, Perez-Morga D, Schtickzelle N, Michels PA. Turnover of glycosomes during life-cycle differentiation of Trypanosoma brucei. Autophagy 4(3), 294-308 (2008).

11. Alvarez VE, Kosec G, Sant'anna C, Turk V, Cazzulo JJ, Turk B. Autophagy is involved in nutritional stress response and differentiation in Trypanosoma cruzi. J. Biol. Chem. 283(6), 3454-3464 (2008).

12. Leveque MF, Berry L, Cipriano MJ, Nguyen HM, Striepen B, Besteiro S. Autophagy-related protein ATG8 has a noncanonical function for apicoplast inheritance in Toxoplasma gondii. MBio 6(6), e01446-15 (2015).

13. Proto WR, Jones NG, Coombs GH, Mottram JC. Tracking autophagy during proliferation and differentiation of Trypanosoma brucei. Microb. Cell 1(1), 9-20 (2014).

14. Brennand A, Rico E, Rigden DJ, van der Smissen P, Courtoy PJ, Michels PA. ATG24 represses autophagy and differentiation and is essential for homeostasy of the flagellar pocket in Trypanosoma brucei. PLoS ONE 10(6), e0130365 (2015).

15. Li FJ, He CY. Acidocalcisome is required for autophagy in Trypanosoma brucei. Autophagy 10(11), 1978-1988 (2014). 
16. Galluzzi L, Pietrocola F, Levine B, Kroemer G. Metabolic control of autophagy. Cell 159(6), 1263-1276 (2014).

17. Lamour N, Riviere L, Coustou V, Coombs GH, Barrett MP, Bringaud F. Proline metabolism in procyclic Trypanosoma brucei is down-regulated in the presence of glucose. J. Biol. Chem. 280(12), 11902-11910 (2005).

18. Li FJ, Xu ZS, Soo AD, Lun ZR, He CY. ATP-driven and AMPK-independent autophagy in an early branching eukaryotic parasite. Autophagy 13(4), 715-729 (2017).

19. Nishida Y, Arakawa S, Fujitani K et al. Discovery of Atg5/Atg7-independent alternative macroautophagy. Nature 461(7264), 654-658 (2009).

20. Foldvari-Nagy L, Ari E, Csermely P, Korcsmaros T, Vellai T. Starvation-response may not involve Atg1-dependent autophagy induction in non-unikont parasites. Sci, Rep, 4, 5829 (2014). 